\title{
The use of simulation for training teamwork skills in health care: how low can you go?
}

\author{
J M Beaubien, D P Baker
}

Qual Saf Health Care 2004;13(Suppl 1):i51-i56. doi: 10.1136/qshc.2004.009845

High fidelity simulation has become a popular technique for training teamwork skills in high risk industries such as aviation, health care, and nuclear power production. Simulation is a powerful training tool because it allows the trainer to systematically control the schedule of practice, presentation of feedback, and introduction (or suppression) of environmental distractions within a safe, controlled learning environment. Unfortunately, many within the training community have begun to use the terms simulation and high fidelity simulation almost synonymously. This is unfortunate because doing so overemphasises the instructional technology to the detriment of more substantive issues, such as the training's goals, content, and design. It also perpetuates several myths: simulation fidelity is unidimensional, or higher levels of simulation fidelity lead to increased training effectiveness. The authors propose a typology of simulation fidelity and provide examples of how the different classes of simulation have been successfully used to train teamwork skills in high risk industries. Guidelines are also provided to maximise the usefulness of simulation for training teamwork skills in health care.

See end of article for authors' affiliations

Correspondence to: J M Beaubien, Industrial and Organizational Psychologist, Aptima Inc., 103015 th Street NW, Suite 300, Washington, DC 20005, USA;

jbeaubien@aptima.com
$\mathrm{R}$ ecent years have witnessed tremendous advances in computing power, networking technology, robotics, and artificial intelligence. These advances have fuelled the development of high fidelity simulators for training teamwork skills in aviation, health care, the military, and nuclear power. High fidelity simulation is particularly popular among trainees who assume that because it replicates the "look and feel" of the actual work environment, it will also provide an efficient and valuable learning experience. In fact, this belief has become so pervasive that many within the training community have begun to use the terms simulation and high fidelity simulation almost synonymously.

This is unfortunate for several reasons. Firstly, although it can be a valuable training tool, high fidelity simulation is only one of many tools for training teamwork skills. Secondly, the published literature has yet to reveal a direct relationship between the level of simulation fidelity and training effectiveness. In fact, numerous studies have demonstrated that it is possible to train teamwork related knowledge, skills, and attitudes using cost effective alternatives. ${ }^{12}$ Finally, when training programmes are designed properly, the level of simulation fidelity becomes somewhat less important. ${ }^{3}$

Up to this point, we have referred to simulation fidelity as a unidimensional concept. As a general rule, people who adopt this perspective tend to believe that simulation can be classified as either low or high fidelity. At one time or another, many within the training community (ourselves included) have adopted this perspective. However, during the course of our research, we have come to believe that this perspective is simplistic. Moreover, we believe that it overemphasises the instructional technology to the detriment of more substantive issues, such as the training's goals, content, and design.

The primary objective of this paper is to dispel the myth that simulation fidelity is unidimensional. Our secondary objective is to remind the reader that when a training programme is properly designed, the level of simulation fidelity becomes somewhat less important. Drawing on over two decade's of research from high risk industries, we review the major classes of simulation that have been used for training teamwork skills, the evidence supporting their effectiveness, and their strengths and weaknesses. Our third and final objective is to provide a series of guidelines to help trainers maximise the usefulness of simulation for training teamwork skills in health care.

\section{BASIC DEFINITIONS OF KEY CONCEPTS}

Before describing how simulators can and should be used to train teamwork skills, it is necessary to define basic terms such as team, teamwork, and teamwork skills. A team is defined as a group of two or more individuals who perform some work related task, interact with one another dynamically, have a shared past, have a foreseeable shared future, and share a common fate. ${ }^{4}$ Examples include operating room, labour and delivery, and emergency room teams.

One of the defining characteristics of a team is that the team members must interact with one another to successfully perform the team task. Therefore, teamwork is defined as those behaviours that facilitate effective team member interaction. Common examples include communication, situational monitoring, and decision making. Although the required teamwork behaviours will vary depending on the team's task, recent research suggests that five teamwork behaviours tend to occur with extreme regularity. The so-called big five teamwork behavioursteam leadership, team orientation, mutual 
performance monitoring, back up behaviours, and adaptability-have been observed in virtually all types of teams. ${ }^{5}$

Finally, teamwork skills refer to competencies that individual team members must possess in order to perform the necessary teamwork behaviours. Technically speaking, these competencies can be classified into three major groups: knowledge, skills, and attitudes. Teamwork knowledge refers to factual information that the team members must possess, such as information about the team's mission, or information about the roles and responsibilities of individual team members. Teamwork skills refer to the learned capacity to perform some type of task, such as being able to communicate clearly, concisely, and using the proper phraseology. Unlike knowledge, skills typically include a physical component that must be developed through practice and feedback. Finally, teamwork attitudes refer to mental states that influence the team members to behave in a particular way. Example attitudes include one's belief in the importance of teamwork, and one's preference for working in a team environment. ${ }^{6}$ Depending on the goals of training, the simulation may be designed to improve any combination of team members' knowledge, skills, and attitudes.

\section{SIMULATION AND SIMULATION FIDELITY}

At this point, the reader should have a basic understanding of teams, teamwork, and teamwork skills. However, before we can begin to explore the relationship between simulation fidelity and team training effectiveness, it is necessary for us to define what we mean by the terms simulation and simulation fidelity. Generally speaking, a simulation (or a simulator) is a device that attempts to recreate characteristics of the real world. There are many reasons for using simulation during training. For example, simulation allows the trainer to carefully control the learning environment by altering schedules of practice, introducing feedback, and introducing (or suppressing) environmental distractions, depending on the goals of training.

\section{DISPELLING MYTHS ABOUT SIMULATION AS A TRAINING TOOL}

In training parlance, the term simulation fidelity has traditionally been defined as the degree to which the simulator replicates reality. ${ }^{7}$ Using this definition, simulators are labelled as either low or high fidelity depending on how closely they represent the real system. For example, personal computer (PC) based flight simulators have traditionally been labelled low fidelity, while full scale simulators that realistically simulate an aircraft's visual, auditory, and motion cues have been traditionally labelled high fidelity. Unfortunately, this deceptively simple definition perpetuates the myth that simulation fidelity is a unidimensional concept. As we shall soon see, there is credible evidence to suggest that fidelity is multi-dimensional.

The nature of simulation fidelity has been hotly debated for over two decades. During that time, countless dimensions of simulation fidelity have been proposed. ${ }^{7-10}$ Many of these dimensions have been based on the simulators' technical characteristics. Although these may be important to the engineers who design and build simulations, they are somewhat less useful for the instructional designers who develop training programmes that incorporate simulation to varying degrees. Moreover, technology based typologies invariably become outdated as technologies evolve over time.

One of the most promising typologies of simulator fidelity was proposed by Rehmann and his colleagues (see fig 1 ). ${ }^{10}$ This typology is particularly useful because it is based on the trainer's perspective. Their first dimension, equipment fidelity, concerns the degree to which the simulator duplicates the appearance and feel of the real system. For example, a simulator that realistically mimics the layout of an aircraft cockpit could be described as high in equipment fidelity. Their second dimension, environment fidelity, concerns the extent to which the simulator duplicates motion cues, visual cues, and other sensory information from the task environment. For example, a flight simulator could be defined as high (or low) on environment fidelity, depending on whether the motion and video cues were turned on (or off). Their third dimension, psychological fidelity, concerns the degree to which the trainee perceives the simulation to be a believable surrogate for the trained task. Alternatively, it could be defined as the match between the trainee's performance in the simulator and the real world. For example, a PC based flight simulator could be defined as high in psychological fidelity if the trainees temporarily suspend disbelief and interact much as they would in the real world. ${ }^{10}$

Although the three fidelity components are inter-related, psychological fidelity is generally considered to be the most essential requirement for team training. ${ }^{27}$ Without temporarily suspending disbelief, trainees are unlikely to behave in the simulation as they would in the real world. As a result, the training will have little application to the post-training environment. Previous research suggests that psychological fidelity can be maximised by developing scenarios that mimic the task demands of the real system. Technology that simulates the environmental or equipment characteristics can increase the psychological fidelity of well designed training scenarios, but cannot compensate for poorly designed ones. ${ }^{3}$

On the surface, the question of whether fidelity is unidimensional or multi-dimensional may seem like a purely academic issue. However, it has profound implications for the design of team training programmes. The various dimensions of simulation fidelity require trainers to make a series of conscious design choices, the results of which can substantially reinforce or counteract the goals of training. For example, if the primary goal is to maximise the initial learning of teamwork skills, the trainer may decide to minimise distractions by choosing a simulator that is low in environmental fidelity. However, if the primary goal is to maximise the transfer of trained behaviours to the posttraining environment, the trainer may select a simulator that mimics the environmental conditions of the real system. In

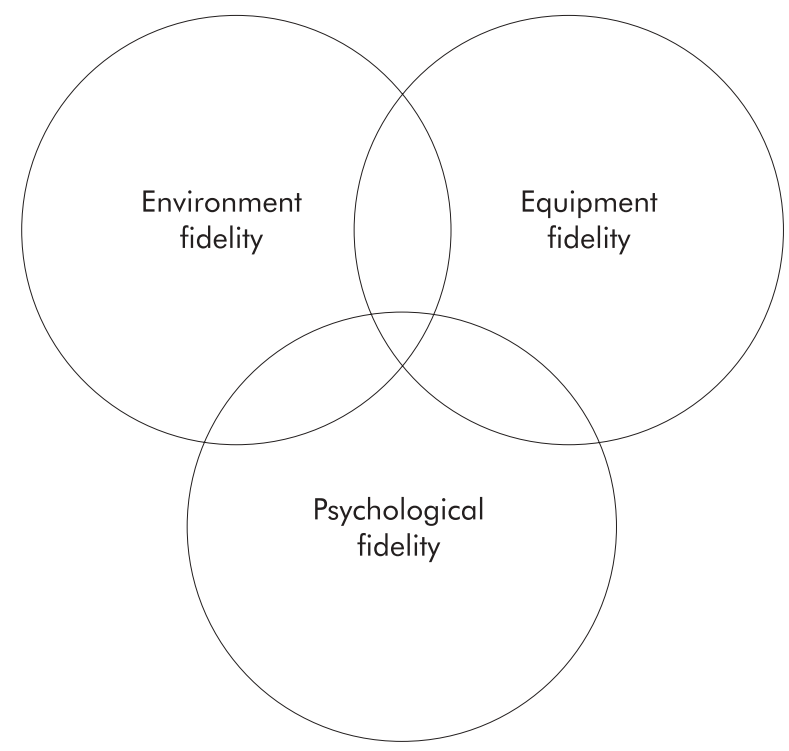

Figure 1 A Typology of simulation fidelity (adapted from Rehmann et al, 1995). ${ }^{10}$ 
reality, training programmes typically have multiple goals, such as to improve immediate learning, long term transfer, and patient safety. Therefore, it is essential that simulation's overall fidelity configuration-equipment, environment, and psychological fidelity-be carefully chosen to reinforce these goals. More often than not, the training will require different levels of fidelity during different exercises at different points throughout the training curriculum.

\section{TYPES OF SIMULATION AND THEIR USE IN TEAM TRAINING}

Over the years, various forms of simulation have been used to train teamwork related knowledge, skills, and attitudes. An early review of the training literature suggested that simulators could be categorised into six broad categories: psychological trainers, familiarisation trainers, part task trainers, vehicle trainers, full mission simulators, and augmentation devices. ${ }^{9}$ However, changing technology has blurred the distinctions among these categories. Based on our own experience, we have proposed a three category typology of simulation technology. Although these are not the only forms of simulation used for training teamwork related competencies, they are perhaps the most common approaches. After providing an overview of each technology, we describe its fidelity configuration. We then review the evidence supporting their effectiveness, as well as their strengths and weaknesses (see table 1).

\section{Case studies and role plays}

Case studies and role plays are two very basic forms of simulation that use fictional examples of team performance to reinforce the trained material. Most case studies require nothing more than paper and pencil. They typically include background information about the event, a synopsis of the team's behaviour during the event, a description of the event's outcome, and a justification as to why the team's performance was particularly effective or ineffective. During a case study, trainees review the factual concepts that they have learned, and discuss how these concepts apply to the fictional example. In addition, the trainees are usually asked how they might act differently if they suddenly found themselves in a similar position. However, there is no attempt to re-enact the event. As a result, case studies are well suited to reinforcing factual knowledge and developing positive attitudes towards the importance of teamwork. Role plays are a slightly more advanced form of case study. Rather than simply describing what they might have done differently, the trainees re-enact the event, or how they would have handled things differently, without the use of props. Role plays are well suited to developing positive attitudes towards the importance of teamwork, reinforcing factual knowledge about teamwork concepts, and-to a lesser extent—developing teamwork skills.

Using the multi-dimensional definition of simulation described earlier, case studies and role plays can be described as low in equipment fidelity, low in environmental fidelity, and low to medium in psychological fidelity. Given their low overall fidelity configuration, many have questioned their effectiveness for training teamwork skills in high risk industries. However, case studies and role plays have been successfully used to train teamwork related attitudes, knowledge, and skills in aviation, ${ }^{11}$ health care, ${ }^{12}$ and corrections. ${ }^{13}$ Case studies and role plays have several strengths. For example, they can be developed and implemented with minimal resource investment. This is particularly important if the training is to be deployed on a large scale, such as throughout the entire military heath care system. Another advantage is that case studies and role plays are usually well received by trainees. This is important, because poor reactions can undermine the credibility of training. Not surprisingly, case studies and role plays also have their weaknesses. For example, they provide only limited opportunities to practice behavioural skills. Moreover, if not properly implemented, they can produce a backlash among certain sub-groups of trainees. ${ }^{11}$

\section{Part task trainers}

Part task trainers can take many forms-such as standardised patients, endotracheal intubators, and simulated anaesthesiology machines-but all follow the same basic principle. The training is designed to segment a complex task into its main components. During the training, the trainees learn each individual subtask to a pre-specified level of competency. As each subtask is mastered, another subtask is added, and both subtasks are practiced together. The addition of subtasks continues until the entire skill has been learned to proficiency. ${ }^{8}$ For example, many commercial airline pilots learn to programme the airplane's flight management computer using a stand alone control panel. The training provides guidance on navigating the flight management computer's menu structure, selecting navigational waypoints, constructing a primary flight route, selecting an alternate flight route, and switching between the two routes.

Using the multi-dimensional definition of simulation described earlier, part task trainers can be described as medium in equipment fidelity, low to medium in environmental fidelity, and medium in psychological fidelity. Part task trainers have been used in health care to teach technical skills such as endotracheal intubation, laparoscopic surgical procedures, and the proper application of cricoid pressure during anaesthesia induction. Part task trainers have also been used to train teamwork related skills for military and civilian pilots using PC based flight simulators. ${ }^{2}$ Part task trainers have a number of strengths. For example, they allow trainees to practice their technical and teamwork skills to a pre-defined standard of competence without being distracted by irrelevant information. ${ }^{14}$ They are also a portable and cost effective means of managing the organisation's limited training budget. For example, some airlines distribute part task trainers to their pilots on CD-ROM. The pilots can practice the materials at home prior to beginning their formal training at the company's training facility. Doing so allows the airline to devote their costly, full mission simulators to more complex training issues. Nevertheless, part task trainers

Table 1 A summary of simulation based training in high risk industries

\begin{tabular}{|c|c|c|c|}
\hline Simulation type & Teamwork competencies & Primary strengths & Primary weaknesses \\
\hline Case studies/role plays & Knowledge, attitudes & $\begin{array}{l}\text { Low cost, positive trainee } \\
\text { reactions }\end{array}$ & $\begin{array}{l}\text { Few opportunities for skills } \\
\text { practice }\end{array}$ \\
\hline Part task trainers & Knowledge, skills & $\begin{array}{l}\text { Low cost, distraction free } \\
\text { environment }\end{array}$ & $\begin{array}{l}\text { No opportunity for dual } \\
\text { task practice }\end{array}$ \\
\hline Full mission simulations & Knowledge, skills & $\begin{array}{l}\text { Can simulate rare (but critical) } \\
\text { tasks in a safe environment }\end{array}$ & $\begin{array}{l}\text { High cost, currently limited } \\
\text { to a few medical specialties }\end{array}$ \\
\hline
\end{tabular}


do have their limitations. The primary limitation is that they do not allow for dual task practice, which inhibits the development of time or resource sharing skills. ${ }^{15}$ However, if part task trainers are used to supplement full mission simulators, this becomes less of an issue.

\section{Full mission simulations}

Full mission simulations are designed to simulate a complex task-such as flying an airplane from takeoff to landingwith all the environmental complexities that go along with it. When used for training teamwork related skills, full mission simulations usually begin with a pre-briefing. During the briefing, the team members discuss their mission, delineate roles and responsibilities, identify likely problems, and establish backup plans for resolving these problems. Next, the team performs the simulated mission, which is usually directed by an instructor who uses a standardised script to manipulate the simulator parameters. Depending on the type of team, the mission may be practiced several times, with the trainees rotating through the various mission roles. Once the simulation is complete, the team members participate in a post-training debrief to identify the lessons that they have learned. In many cases, videotaped examples are used to identify examples of particularly effective and ineffective teamwork.

Using the multi-dimensional definition of simulation described earlier, full mission simulation can be described as high in equipment fidelity, medium to high in environmental fidelity, and high in psychological fidelity. During the past two decades, full mission simulators have been used to successfully train teamwork related knowledge, skills, and attitudes in aviation, ${ }^{16}$ health care ${ }^{17}$ the military, ${ }^{18}$ and nuclear power. ${ }^{19}$ Full mission simulation has a number of strengths. For example, it allows trainees the opportunity to practice their trained skills under realistic conditions, and to observe the consequences of their actions/inactions-such as the death of a patient or the crash of an airplane-in a safe environment. Full mission simulation also allows trainees to prepare for extremely rare, but highly critical emergency situations that would be impossible to train otherwise. Nevertheless, full mission simulation does have its weaknesses. The primary weakness is its cost. Even with recent reductions in technology costs, the personnel costs associated with developing, implementing, and maintaining full mission simulations makes them impractical for many organisations. Moreover, because many of them are tailored to specialty areas such as anaesthesia and surgery, they may be less useful for other disciplines. This is not to say all disciplines cannot benefit from full mission simulation training, it is just that not every discipline has commercially available full mission simulation at this time. In addition, unless the organisation allows the trainees to practice their teamwork related skills prior to participating in the full mission simulation, the trainees may be overwhelmed by the environmental distractions, stress, and time pressure.

\section{MAXIMISING THE USEFULNESS OF SIMULATION IN HEALTHCARE TEAM TRAINING}

The published literature on simulation as a training tool is extremely fragmented, and many critical training issues remain unexplored. For example, during the course of our research, we were unable to locate any studies that used multiple types of simulation to train identical teamwork related competencies. As a result, it is impossible to quantify the relative effectiveness of different types of simulation for given set of criteria. However, we were able to identify a number of principles for maximising the effectiveness of simulation as a training tool. Unfortunately, many of these principles were more scientific than practitioner oriented. In the following paragraphs, we propose a series of practical guidelines that instructional designers can use to maximise their limited training resources.

Carefully tailor your training needs, goals, content, and evaluation measures to reinforce one another Developing a training programme is an extremely complex task. Therefore, it is essential that the training begins with a comprehensive needs analysis. ${ }^{80}$ This information should then be used to specify the goals of training, the content of the simulation, and the content of the post-training evaluation. If the training is designed properly, the linkages among these elements should be readily apparent. ${ }^{3}$ However, because training design is an iterative process, these linkages can become broken. One technique for maintaining these linkages is to use a programme audit database, which ensures a direct correspondence between training needs, goals, content, and evaluation. The programme audit database is a major component of many commercial airline training programmes, and can be created using off the shelf database software.

\section{Use case studies and role plays to train teamwork related knowledge and attitudes}

The learning process occurs in a series of discrete stages. During the earliest stages of learning, trainees acquire factual knowledge about the content area (what is involved in performing the task). With sufficient practice, factual knowledge becomes compiled into relatively automatic skills (how to perform the task), which require less conscious attention. At the highest stages of skill acquisition, trainees develop tactical knowledge (when to perform the task) and metacognitive skills (how to self-regulate one's performance). ${ }^{21}$ However, proceeding through these successive stages requires a substantial investment of training resources. Therefore, we recommend that case studies and role plays be used as the primary simulation technology for training attitudes toward the importance of teamwork and knowledge of teamwork concepts. These simulation techniques are generally cost effective, can be deployed on a large scale, and are unlikely to overwhelm the trainees during the earliest stages of learning.

Use part task trainers to train teamwork related skills to the point of over-learning

After mastering the background knowledge and attitudes, it is essential that trainees practice their teamwork skills. We recommend the use of part task trainers—such as simulated patients, and PC based anaesthesiology simulators-so that the trainees can practice their teamwork skills to the point of over-learning. Just as in cardiopulmonary resuscitation training, the goal of over-learning is to ensure that the task can be performed properly every time. Part task trainers are particularly well suited to this, because they are relatively portable and cost effective. As a result, they can be deployed on a large scale.

Use full mission simulators to hone teamwork related skills under conditions of ambiguity, time pressure, and stress

In many cases, trainers will not have access to full mission simulations. If they do, we recommend that they use full mission simulations almost exclusively to hone the trainees' already learned teamwork skills. As noted earlier, part task trainers are an effective simulation tool, but they do not provide trainees with the opportunity to practice their time or resource sharing skills. Therefore, we recommend that trainers consider using the scaffolding technique during full mission simulation. The scaffolding technique involves having an experienced staff member initially take over some of the simulated task requirements. Over time, the staff 
member gradually withdraws from the task, thereby leaving the team to perform on their own. Scaffolding can prevent the team from becoming overwhelmed during their first few full mission simulations. ${ }^{22}$

\section{Use post-simulation debriefings to reinforce the lessons learned during training}

There is an old saying that "practice makes perfect". In reality, practice makes behaviour more or less permanent. Perfection can only be achieved through practice with feedback. As a result, many instructional designers use post-simulation debriefings to help the team members learn from their mistakes and develop action plans for future selfdevelopment. Some debriefs may be instructor led; others are team led. Likewise, some debriefs employ videotape-to point out specific examples of effective and ineffective teamwork behaviour during the simulation-while others do not. Our research has shown that no one type of debrief is clearly superior than the others. ${ }^{23}$ Therefore, we recommend that, at a minimum, some form of post-simulation debrief be used to identify the lessons that were learned, and to generate strategies for team self-development.

\section{Training is not a one time event, so plan accordingly}

We view training as a learning process, not as a one time event. Like every skill, teamwork competencies decay without periodic reinforcement and practice. Therefore, instructional designers need to carefully conserve their limited training resources so that they can include the most appropriate combination of awareness training (which focuses on knowledge and attitudes), skills practice, and recurrent skills maintenance, depending on the their unique training goals. For many organisations, the use of multiple training strategies may represent a cultural shift.

\section{CONCLUSIONS AND LESSONS LEARNED}

In recent years, high fidelity simulation has become an increasingly popular tool for training teamwork skills in high risk industries. Although we do not doubt its usefulness as a training tool, we are disturbed by the tendency to equate the term simulation with high fidelity simulators. We believe that this is unfortunate, because other types of simulation, such as case studies, role plays, and part task trainers, have an established base of research to support their effectiveness for training teamwork related attitudes, knowledge, and skills. Although there is a tendency to believe that more fidelity is always better, the published research does not support this conclusion. Specifically, we were unable to identify any studies that found a direct correlation between the level of simulation fidelity and training related outcomes, such as learning, transfer, and safety. Like any other tool, the effectiveness of simulation technology depends on how it is used. ${ }^{24}$

In the final analysis, the choice of simulation depends on a number of factors, such as the training needs, the available resources, and the number of people to be trained. In a perfect world you would provide as much training as necessary to maintain the margin of safety. For example, if trainees required $50 \mathrm{~h}$ of teamwork skills training per year$10 \mathrm{~h}$ of lecture and case studies, $30 \mathrm{~h}$ of part task trainers, and $10 \mathrm{~h}$ of full motion simulation-that is what you would provide. More often than not, the training budget is underfunded, the training staff are overworked, and the time frame is unrealistically short. Therefore, we recommend that trainers maximise their training resources by leveraging low fidelity simulations to the greatest extent possible. When properly designed, these alternatives to full mission simulation can be a cost effective means for training teamwork related knowledge, skills, and attitudes.

\section{Key messages}

- The three major components of simulation fidelity are equipment fidelity, environment fidelity, and psychological fidelity. Of the three, psychological fidelity is the most important for teamwork skills training.

- There is no direct relationship between the level of simulation fidelity and teamwork training effectiveness.

- High fidelity simulations can enhance the perceived realism of well designed team training programmes, but cannot compensate for poorly designed ones.

- Instructional designers need to carefully tailor the simulation's overall fidelity configuration to match the goals and content of team training.

- Most team training programmes will require different levels of simulation fidelity throughout the training curriculum.

\section{Areas for further research and development}

- The various dimensions of simulator fidelity have all been developed based on theoretical and practical considerations. Research is needed to empirically validate these dimensions and to assess the extent to which they overlap.

- To date, no studies have compared different types of simulation for training identical teamwork related competencies. Therefore, it is impossible to quantify the relative effectiveness of different types of simulation.

- Simulation based training has resulted in improved attitudes toward the importance of teamwork and improved teamwork behaviours. However, the link to improved patient safety needs to be more clearly demonstrated.

- Because simulation based training can be extremely expensive, trainers need practical guidelines on how to calculate the return on their training investment (ROI).

In the title of this paper, we asked "How low can you go?" when it comes to simulation fidelity in teamwork skills training. As we have shown, low cost technologies, such as case studies, role plays, and part task trainers, have been effectively used to train teamwork related knowledge, skills, and attitudes in several high risk industries. Although trainees and instructional developers may prefer the "bells and whistles" of full mission simulators, we implore them to at least explore the use of lower fidelity alternatives, especially during the earliest phases of teamwork skill acquisition. After all, effective training is clearly not synonymous with full mission simulation.

\section{Authors' affiliations}

J M Beaubien, D P Baker, American Institutes for Research, Washington DC, USA

\section{REFERENCES}

1 Baker D, Prince C, Shrestha L, et al. Aviation computer games for crew resource management training. International Journal of Aviation Psychology 1993;3:143-156. 
2 Jentsch F, Bowers CA. Evidence for the validity of PC-based simulations in studying aircrew communication. International Journal of Aviation Psychology 1998;8:243-260

3 Oser R, Cannon-Bowers JA, Salas E, et al. Enhancing human performance in technology-rich environments: guidelines for scenario-based training. Human/Technology Interaction in Complex Systems 1999;9:175-202.

4 Salas E, Cannon-Bowers JA, Blickensderfer EL. Team performance and training research: emerging principles. Journal of the Washington Academy of Sciences 1993:83:81-106.

5 Salas E, Sims DE, Burke CS. Is there a "big five" in teamwork? Paper presented at the 19th Annual Meeting of the Society for Industrial and Organizational Psychology. Chicago, IL, 2004.

6 Cannon-Bowers JA, Salas E. Teamwork competencies: the interaction of team member knowledge, skills, and attitudes. In: O'Neil HF Jr, ed. Workforce readiness: competencies and assessment. Mahwah, NJ: Lawrence Erlbaum Associates, 1997:151-174.

7 Alessi S. Simulation design for training and assessment. In: $\mathrm{O}^{\prime} \mathrm{Neil} \mathrm{H} \mathrm{Jr}$, Andrews DH, eds. Aircrew training and assessment. Mahwah, NJ: Erlbaum, 2000:197-222.

8 Goldstein I. Training in organizations: needs assessment, development, and evaluation. Pacific Grove, CA: Books/Cole, 1993.

9 Farrow D. Reducing the risks of military aircrew training through simulation technology. Performance and Instruction 1982;21:13-18.

10 Rehmann A, Mitman R, Reynolds M. A handbook of flight simulation fidelity requirements for human factors research. Technical Report No. DOT/FAA/ CT-TN95/46. Wright-Patterson AFB, OH: Crew Systems Ergonomics Information Analysis Center, 1995.

11 Helmreich RL, Merritt AC, Wilhelm JA. The evolution of crew resource management training in commercial aviation. International Journal of Aviation Psychology 1999;9:19-32.

12 Morey J, Simon R, Jay G, et al. Error reduction and performance improvement in the emergency department through formal teamwork training: evaluation results of the MedTeams project. Health Services Research 2003;37:1553-81.

13 Crichton M, Flin R, Rattray W. Training decision makers - tactical decision games. Journal of Contingencies and Crisis Management 2000;8:208-17.
14 Ewy G, Felner J, Juul D, et al. Test of a cardiology patient simulator with students in fourth-year electives. Journal of Medical Education 1987;62:738-43.

15 Bowers CA, Braun C, Morgan BB. Team workload: its meaning and measurement. In: Brannick MT, Salas E, Prince C, eds. Team performance measurement and assessment. Mahwah, NJ: Erlbaum, 1997:85-108.

16 Butler RE. LOFT: full mission simulation as crew resource management training. In: Wiener EL, Kanki BG, Helmreich RL, eds. Cockpit resource management. San Diego: Academic Press, 1993:231-59.

17 Gaba DM. Anesthesiology as a model for patient safety in health care. BMJ 2000;320:785-8.

18 Stretton M Johnston J, Cannon-Bowers JA. Conceptual architecture for embedded team training management. Human/Technology Interaction in Complex Systems 1999;9:87-120.

19 Gaddy C, Wachtel J. Team skills training in nuclear power plant operations. In: Swezey R, Salas E, eds. Teams: their training and performance. Norwood, NJ: Ablex, 1992:379-396.

20 Swezey RW, Owens JM, Bergondy ML, et al. Task and training requirements analysis methodology (TTRAM): an analytic methodology for identifying potential training uses of simulator networks in teamwork-intensive task environments. Ergonomics 1998;41:1678-97.

21 Kraiger K, Ford JK, Salas E. Application of cognitive, skill-based and affective theories of learning to new methods of training evaluation. Journal of Applied Psychology 1993:78:311-28.

22 Means B, Salas E, Crandall B, et al. Training decision makers for the real world. In: Klein GP, Orasanu J, Calderwood R, et al. Decision making in action: models and methods. Norwood, NJ: Ablex, 1995.

23 Beaubien JM, Baker DP. Post-training feedback: the relative effectiveness of team- versus instructor-led debriefings. Proceedings of the 47th Annual Meeting of the Human Factors and Ergonomics Society. Santa Monica, CA: Human Factors and Ergonomics Society, 2003:2033-6.

24 Salas E, Bowers CA, Rhodenizer L. It is not how much you have but how you use it: toward a rational use of simulation to support aviation training. International Journal of Aviation Psychology 1998;8:197-208. 